\title{
A patient with rectum cancer, and subcutaneous and pulmonary metastases
}

\section{Case report}

A 27-year-old male patient was admitted to Dokuz Eylul University Hospital (Izmir, Turkey) with complaints of constipation and weight loss for a period of 4 months. His history was not significant, except for the early age at which he started to smoke, resulting in a smoking habit of one pack of cigarettes per day for 12 years. There was no history or evidence of cancer in any of his close relatives.

A rectosigmoidoscopic examination showed an ulcerated vegetating mass of $\sim 3 \mathrm{~cm}$ in the anorectal region. A histopathological sample of the mass is shown in figure 1.

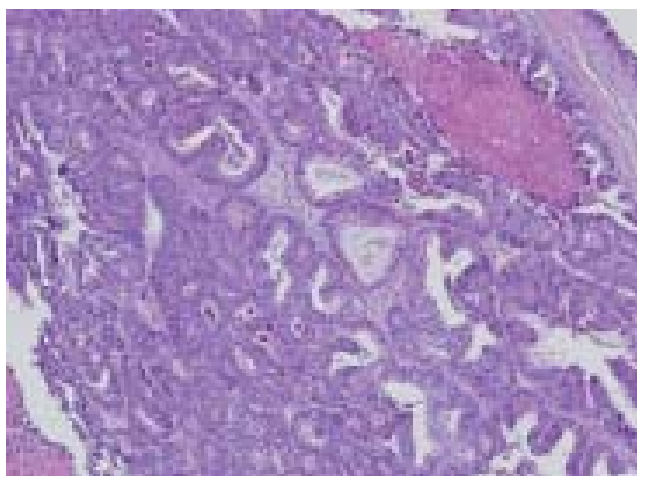

Figure 1

Biopsy sample from rectal mass.

\section{Task 1}

Interpret the histological findings from the rectal mass.
A. Alacacioglu
D. Solmaz
I. Oztop ${ }^{1}$
0. Tarhan $^{1}$
A. Yaren ${ }^{1}$
S. Yassin ${ }^{2}$
S. Sokmen ${ }^{3}$
A. Kargi ${ }^{4}$
A. Akkoclu ${ }^{5}$
U. Yilmaz ${ }^{1}$

${ }^{1}$ Institute of Oncology, and Depts of ${ }^{2}$ Internal Medicine, ${ }^{3}$ General Surgery, ${ }^{4}$ Pathology, and ${ }^{5}$ Chest Disease, Faculty of Medicine, Dokuz Eylul University, Izmir, Turkey.

\author{
Correspondence: \\ A. Alacacloğlu \\ Huzur mah. Acelya sok. No: 26/7 \\ Narlidere, 35320 \\ Izmir \\ Turkey \\ Fax: 902324123719 \\ E-mail: \\ ahmet.alacacioglu@deu.edu.tr
}




\section{Answer 1}

Histopathological examination identified an adenocarcinoma.

Immunohistochemical staining for CDX2 and cytokeratin 20 were positive. Staging procedures, including thoracoabdominal computerised tomography (CT), were negative for distant metastases. The tumour was removed by low anterior resection. Histopathological evaluation of the surgical sample confirmed the diagnosis of adenocarcinoma and the pathological stage was reported as T3NO. Post-operative thoracoabdominal CT was normal, as was the carcino-embryonic antigen (CEA) level of $2.3 \mathrm{IU}$ per $\mathrm{mL}$ (upper limit of normal (ULN) 4 IU per $\mathrm{mL}$ ).

Adjuvant chemotherapy was initiated 3 weeks after surgery, consisting of 12 cycles of LV5FU2 with infusional 5-fluorouracil, preceded by high-dose folinic acid (De Gramont regimen) [1].

After chemotherapy, thoracic CT identified two metastatic lesions in the right lung. Serum CEA remained under the ULN, but showed a slight increase to a level of $3.6 \mathrm{IU}$ per $\mathrm{mL}$. Therefore, a regimen consisting of irinotecan and raltitrexed was initiated as second-line chemotherapy. This regimen, which was given for four cycles, was stopped because of progression of the lung metastasis. As oxaliplatin is not available in Turkey, single-agent chemotherapy with capecitabine was started as third-line treatment.

After the second cycle of capecitabine, the metastasis in the right lung progressed and a new right hilar mass of $6 \mathrm{~cm}$ was found (figure 2). At the same time, a 6-cm, red-pink, swollen and painless solitary mass appeared on the right chest wall (figure 3). At this stage, the CEA level was $1.7 \mathrm{IU}$ per $\mathrm{mL}$.

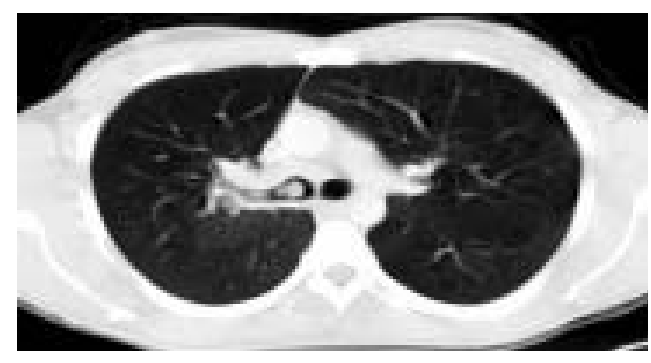

Figure 2

Thoracic CT of the lung metastasis and new right hilar mass after third-line chemotherapy.

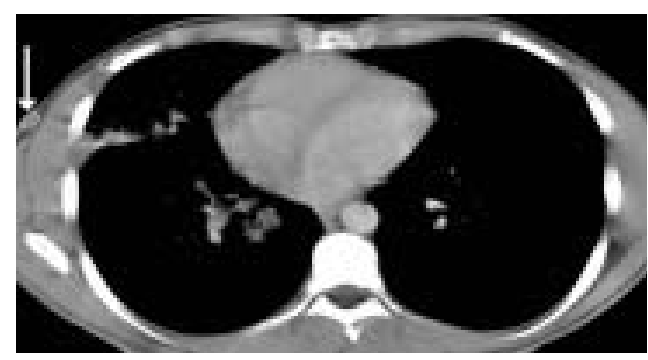

Figure 3

Thoracic CT of the subcutaneous metastasis on the anterior side of the right hemithorax.

A transbronchial biopsy was obtained from the right hilar mass, and the histopathological examination is shown in figure 4 . The subcutaneous lesion was also completely excised, and histopathological findings are shown in figure 5 . 


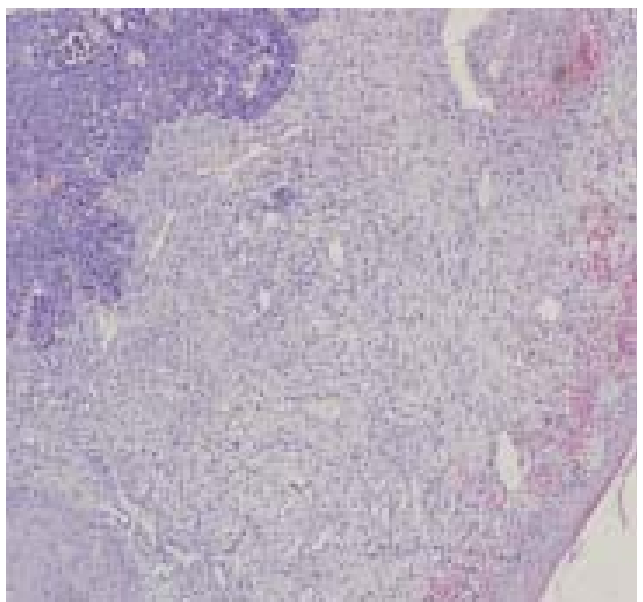

Figure 4

Transbronchial biopsy material taken from the right hilar mass.

\section{Task 2 \\ Interpret the histopathological find- ings from the transbronchial biopsy taken from the right hilar mass.}

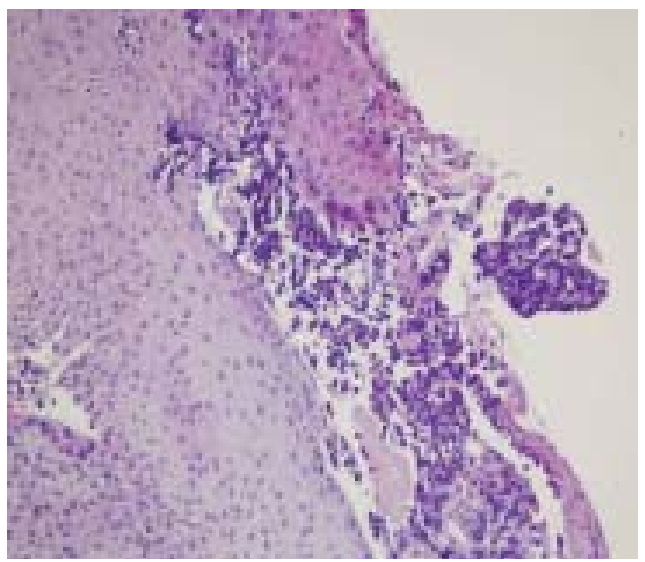

Figure 5

Biopsy sample taken from the subcutaneous metastasis.

\section{Task 3}

Interpret the histopathological findings from the excised subcutaneous lesion.

\begin{abstract}
Answer 2
Histopathological examination diagnosed small-cell lung carcinoma.
\end{abstract}

\section{Answer 3}

Histopathological examination revealed adenocarcinoma.

Immunohistochemical staining was positive for thyroid transcription factor- 1 , and negative for CDX2 and cytokeratin 20. The patient refused any further chemotherapy. External radiotherapy permitted some palliation for airways obstruction, but the patient died at home 2 months after the cessation of chemotherapy.

\section{Discussion}

Cancer patients are at a relatively high risk of developing a secondary primary cancer. However, in the case of colorectal cancer, synchronous and metachronous secondary cancer rates are low. Rates of $0.5-3.0 \%$ have been reported in the current literature, although one study found rates of 4.8 and $9.0 \%$ for synchronous and metachronous tumours, respectively [2].

The mechanisms behind the development of these tumours are not well elucidated, but predisposing risk factors have been previously well defined. These factors include synchronous polyps or cancer, a metachronous tumour history and hereditary non-polyposis colorectal carcinomas [3].

The most commonly reported secondary cancers are renal cell carcinoma and breast cancer (2.9-3.8\%), whereas cases of secondary lung cancer are rare [4]. However, lung cancer patients frequently suffer from other tobacco-related cancers. Therefore, there is no established link between lung and colon cancer [5]. In general, secondary primary cancers are observed within 3 years from the initial diagnosis and have a very poor prognosis.

To date, in the literature, there has only been one case of colorectal cancer followed by secondary lung cancer. In this patient, early-stage rectum cancer was treated by low anterior resection alone. Five months later, during follow-up for pneumoconiosis, an early-stage adenocarcinoma was found and treated with a left upper lobectomy [6]. 
In the present case, the interval between the initial diagnosis and the metastasis diagnosis was longer, i.e. 22 months. The first lung lesions were accepted as metastases of the rectal cancer and no histopathological examination was performed. However, the hilar lesion observed later was different from the previously observed peripheral lesions, because it had a rapid course in appearance and an aggressive character. Therefore, given these characteristics and their presence in a heavy smoker, small cell lung cancer (SCLC) was suspected, and then confirmed via histopathological examination and immunohistochemical staining. Treatment consisting of cisplatin plus etoposide chemotherapy was proposed; however, the patient would not accept any further chemotherapy.

Subcutaneous metastases are also uncommon in colorectal cancer (4-5\%) [7]. They are sometimes observed in rectal adenocarcinomas, but not in carcinomas found in other parts of the colon. Subcutaneous metastases from colon carcinomas are generally identified after a 2-year period after removal of the primary tumour. Subcutaneous metastases are typically observed in patients with advanced disease, and are accompanied by an overall survival time of 3-18 months $[8,9]$. In this patient, a rapidly growing subcutaneous metastasis was observed and histopathological examination revealed the relationship with primary rectal adenocarcinoma.

This study presented a patient with rectal adenocarcinoma, which relapsed with distant metastases, who died from a secondary cancer identified as SCLC. The reasons for the development of SCLC are unknown, although it may have been related to smoking history and chemotherapy agents.

In conclusion, in such patients with advanced colorectal cancer, the appearance of new lesions with different characteristics should alert the clinician to the possibility of a different secondary cancer. An awareness of this issue is also important due to the potential impact on the treatment of the patient.

\section{References}

1. de Gramont A, Bosset JF, Milan C, et al. Randomized trial comparing monthly low-dose leucovorin and fluorouracil bolus with bimonthly high-dose leucovorin and fluorouracil bolus plus continuous infusion for advanced colorectal cancer: a French intergroup study. J Clin Oncol 1997; 15: 808-815.

2. Heald RJ. Synchronous and metachronous carcinoma of the colon and rectum. Ann R Coll Surg Engl 1990; 72: 172174.

3. Fante $R$, Roncucci $L, D i$ Gregorio $C$, et al. Frequency and clinical features of multiple tumors of the large bowel in the general population and in patients with hereditary colorectal carcinoma. Cancer 1996; 77: 2013-2021.

4. Fajobi O, Yiu CY, Sen-Gupta SB, Boulos PB. Metachronous colorectal cancers. Br J Surg 1998; 85: 897-901.

5. Levi F, Randimbison L, Te VC, Vecchia C. Second primary cancers in patients with lung carcinoma. Cancer 1999; 86: 186-190.

6. Tsuji $K$, Hori K, Kimura S, et al. Experience with surgeny for synchronous double cancer of rectum and lung detected after long-term follow-up for pneumoconiosis. Kyobu Geka 1996; 49: 1130-1133.

7. Stavrianos SD, Mclean NR, Kelly CG, Fellows S. Cutaneous metastasis to the head and neck from colonic carcinoma. Eur J Surg Oncol 2000; 26: 518-519.

8. Damin DC, Lazzaron AR, Tarta C, Cartel A, Rosito MA. Massive zosteriform cutaneous metastasis from rectal carcinoma. Tech Coloproctol 2003; 7: 105-107.

9. Adani GL, Marcello D, Anania G, et al. Subcutaneous right leg metastasis from rectal adenocarcinoma without visceral involvement. Chir Ital 2001; 53: 405-407. 